\title{
Application of Catastrophe Theory to Draw Resonance
}

\author{
Riichirô CHÛJÔ and Koichi TsUYAMA \\ Department of Polymer Chemistry, Tokyo Institute of Technology, \\ 12-1 Ookayama 2-chome, Meguro-ku, Tokyo 152, Japan.
}

(Received July 7, 1979)

\begin{abstract}
Catastrophe theory was successfully applied to the analysis of draw resonance phenomena. It was shown that the potential of cusp catastrophe was appropriate to the analysis of oscillating viscoelasticity appearing in the above-mentioned phenemena. Moreover, basic behavior such as stress relaxation is discussed in regard to this potential. In these analyses, no relaxation spectrum is introduced. However, an apparent spectrum can be obtained if the results of stress relaxation are analyzed by a procedure similar to that used in the analysis of linear viscoelasticity. But a spectrum may not always exist even if derived analytically.

KEY WORDS Draw Resonance / Catastrophe Theory / Cusp Catastrophe / Nonlinear Viscoelasticity / Relaxation Spectrum /
\end{abstract}

The theory of linear viscoelasticity has widely been accepted as a tool for describing viscoelastic phenomena of high polymers by the introduction of relaxation or retardation spectrum. ${ }^{1}$ Furthermore, this theory enables us to describe the stress-strain relationship, using the Boltzmann superposition principle, since this theory is very convenient to analyse such phenomena. In spite of this advantage, we have no direct proof that there must be a relaxation spectrum. In other words, the introduction of the spectrum is not inevittable but conventional.

According to recent results in NMR relaxation studies of high polymers both in bulk and in solution, viscoelasticity can be explained as a discrete system with respect to their time constants. In the broadline NMR spectrum of poly(methyl methcacrylate), the ratio of the half width to the whole width between maximum slopes is 1.6 at the room temperature. ${ }^{2}$ This value is close to 1.732 which is the spectrum expected with a Lorentzian shape. This implies that this polymer rapidly fluctuates with a singele correlation time at room temperature. In the pulsed NMR measurement of vulcanized natural rubber, the solid echo is the Fourier transform of the Weibull function in the rubbery region. ${ }^{3}$ Such a solid echo curve cannot be obtained if one introduces any kind of relaxation spectrum. Even in the case of a partially crystalline polymer, the solid echo from the amor- phous region cannot be analyzed with the relaxation spectrum. The mobile region of low-density polyethyelene is a typical example. ${ }^{4}$ The discreteness of the time constants also appears in the highresolution NMR spectrum of the polymer solution. In a trans-decalin solution of a polypropylene the PRFT (partially relaxed Fourier transform) ${ }^{13} \mathrm{C}$ NMR spectrum, there are many peaks corresponding to chemical groups and stereochemical configurations. ${ }^{5}$ The intensity of each peak in the PRFT spectrum recovers exponentially with respect to its thermal equilibrium value with a corresponding discrete time constant. As seen from experimental evidence, no positive proof for the necessity of a relaxation spectrum from NMR experiments could be found. Of course, there are papers in which NMR experiments are analyzed under the assumption of the necessity of a relaxation spectrum. However, these papers are not poor of the significance of this assumption.

There are many works on viscoelasticity of polymers in which nonlinear terms are consedered. A typical treatment ${ }^{6}$ is the use of time-dependent variables instead of constants which are strictly constant in the linear theory. Such an extension can be recognized as a perturbation method in which the nonlinearity is a perturbation to the unperturbed linear viscoelasticity term. This situation is quite different from the nonlinear theory in other fields of 
science such as mathematical ecology, ${ }^{7}$ where the nonlinearity due to interaction between species is introduced as an essential factor.

In this paper we will apply catastrophe theory to nonlinear viscoelasticity in polymers; the distinct features of our treatment are (1) that no relaxation spectrum is required, (2) there is no perturbation theory caused by a nonlinear term, and (3) that our treatment is applicable to oscillating nonlinear phenomena such as draw resonance in melt spinning process. Ishihara and $\mathrm{Kase}^{8}$ and Denn and Fischer ${ }^{9}$ analyzed draw resonance phenomena with only viscosity terms. Though our trestment is different from theirs, we analyze the phenomena by viscoelasticity terms. The arrangement of this paper is as follows; the Maxwell element is, at first, surveyed from the standpoint of dynamical system; this element is, secondly, extended to nonlinear model with the aid of the catastrophe theory; stress relaxation and apparent relaxation spectra derived from the nonlinear model are discussed; the nonlinear model is further extended to the viscoelastic phenomena with oscillation; and finally, draw resonance phenomena are analyzed with the latter model.

\section{DYNAMICAL SYSTEM OF MAXWELL ELEMENT}

The fundamental equation for the Maxwell model of viscoelasticity with stress $\sigma$ and strain $\varepsilon$ is given by,

$$
\mathrm{d} \sigma / \mathrm{d} t=-\sigma / \tau+M_{\mathrm{g}}(\mathrm{d} \varepsilon / \mathrm{d} t)
$$

where $t$ is the time, $\tau$ is the relaxation time, and $M_{\mathrm{g}}$ is the modulus of elasticity. This equation can be rewritten in terms of the following two equations,

$$
\begin{gathered}
d \sigma / \mathrm{d} t=-\sigma / \tau+M_{\mathrm{g}} \beta \\
\mathrm{d} \varepsilon / \mathrm{d} t=\beta
\end{gathered}
$$

where $\beta$ is the rate of strain. In the case of the exponential decay of $\beta$, this quantity may be expressed as

$$
\mathrm{d} \beta / \mathrm{d} t=-\beta / \tau^{\prime}
$$

where $\tau^{\prime}$ is the decay constant for $\beta$. In the simplest experiment, $\varepsilon$ is applied stepwise. In other words, $\beta$ is a form of the Dirac delta function. It may be thought that this type of excitation is inevitably delayed to some extent in practice. Such a delay is expressed in eq 4 in the form of simple exponential decay.

The quantities $\sigma$ and $\beta$ can be solved as functions of time. Eliminating time from these two quantities, we can draw a number of trajectories on $\beta-\sigma$ plane. In Figure 1 are shown tranjectories under conditions in which $M_{\mathrm{g}}=\tau=\tau^{\prime}=1$.

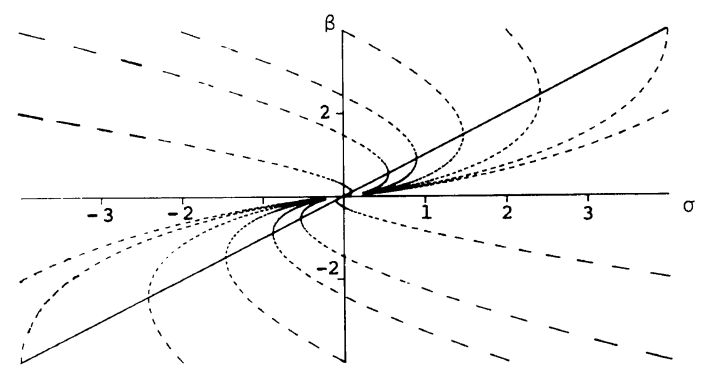

Figure 1. A family of trajectories of the relaxation due to the Maxwell model (2) and eq 4 on $\beta-\sigma$ plane. Selection is made with $M_{\mathrm{g}}=\tau=\tau^{\prime}=1$. Each segment and each spacing separated by segments in the broken lines corresponds to the change of the system in the same interval of time. Solid lines and the abscissa are the minima of pseudo-potentials.

Linear differential equations such as eq 2 and 4 can be summarized in the general form of

$$
\mathrm{d} x / \mathrm{d} t=-f(x)
$$

where $x$ is the representative of $\sigma$ and $\beta$, and $f(x)$ is a function of $x$. The variable $x$ is called a dynamical variable. Defining the pseudo-potential,

$$
V(x)=\int f(x) \mathrm{d} x
$$

of $x$, we can say that the system moves towards a minimum of the pseudo-potential along an appropriate trajectory. The pseudo-potential derived from the right-hand side of eq 2 is,

$$
V(\sigma)=\left(\sigma-M_{\mathrm{g}} \beta \tau\right)^{2} / 2 \tau-M_{\mathrm{g}}^{2} \beta^{2} \tau / 2
$$

and its minimum lies in

$$
\sigma-M_{\mathrm{g}} \beta \tau=0
$$

The solid straight line in Figure 1 corresponds to eq 8 . Similarly, the minimum of the pseudo-potential derived from the right-hand side of eq 4 lies in

$$
\beta=0
$$

This is the axis of abscissa in Figure 1. Each trajectory is, at first, directed to the straight line defined by eq 8 . However, this straight line is not the asymptote of the trajectories. Crossing over eq 8 , 
each trajectory is asymptotically directed to the abscissa in Figure 1, and finally tends to the origin in which both pseudo-potentials take on their minimum values. We can say that the slow relaxation towards the origin of the $\beta-\sigma$ plane is preceded by the rapid relaxation towards eq 8 in the system described by eq 2 and 4 . In the case of the viscoelasticity in liquid-like bodies, this situation becomes important, because of a comparatively small value of $\tau$.

\section{CONSTRUCTION OF CATAST ROPHE MODEL}

The catastrophe theory is a branch of mathematics founded by Thom. ${ }^{10}$ The finite classification theorem of the theory states the classification of topological shapes of the pseudo-potentials when the generalized force is defined by the gradient of pseudo-potential. More precisely, if the number of control variables or parameters such as $\beta$ in eq 2 is less than or equal to four, the topologically different shapes of the possible potentials are classified into only seven types. We must say here that the above statements are not original version of Thom but an alternative appropriate for describing phenomena encountered in natural science.

The linear viscoelasticity described by eq 2 is the behavior occuring in the harmonic pseudo-potential of eq 6 . This means that the nonlinear viscoelasticity is the behavior occuring in an anharmonic pseudopotential. Apart from phenomena such as evolution in which the system changes gradually over a very long time interval compared with the life of a human being, all of the phenomena must be described with the potential whose derivative is positive in the region of very large values of $x$, and negative for very small values of $x$. This is due to the requirement of the stability of system. A quartic function fulfills the above mentioned conditions for the potential and is the simplest among anharmonic potentials. The potential with a quartic form is strictly confined in a topological sense to that of cusp catastrophe according to Thom's theorem. The nonlinear form corresponding to eq 2 is, therefore,

$$
\mathrm{d} \sigma / \mathrm{d} t=-\left(\sigma^{3}-A \sigma-\beta\right) / \tau
$$

This equation is the abstract form in the topological sense. Then, some coefficients and terms are neglected for explicitly. Fro example, the coefficient with the dimension of $\left[\sigma^{-2}\right]$ is neglected in the first term of the right-hand side. Furthermore, if $\sigma$ and $\beta$ approach nonvanishing values of $\sigma_{\mathrm{e}}$ and $\beta_{\mathrm{e}}$, respectively, $\sigma$ and $\beta$ must have the meaning of $\sigma-\sigma_{\mathrm{e}}$ and $\beta-\beta_{\mathrm{e}}$, respectively. The terms $-\sigma_{\mathrm{e}}$ and $-\beta_{\mathrm{e}}$ are dropped also in eq 10. This equation is isomorphous with van der Pol's equation in the theory of electric circuit.

The factor $A$ in the second term of the right-hand side in eq 10 is very important. If the sign of $A$ is confined to be negative, the term, $-\sigma^{3} / \tau$, may be thought to be a perturbation to the term, $A \sigma / \tau$. On the contrary, if the sign of $A$ is positive, the situation is quite different, because the term, $-A / \tau$, loses the meaning of relaxation time.

In Figures $2 \mathrm{a}$ and $2 \mathrm{~b}$ are shown trajectories similar to those in Figure 1 obtained from eq 4 and 10 for $A=1.0$ and -1.0 , respectively. $A=1.0$ and -1.0 are the representatives of $A>0$ and $A<0$, respectively. In both figures, the system changes along a trajectory from the margin of the figures to potential minimum curves, $\sigma^{3}-A \sigma-\beta=0$, which are drawn as solid lines. The system finally tends to the intersections of

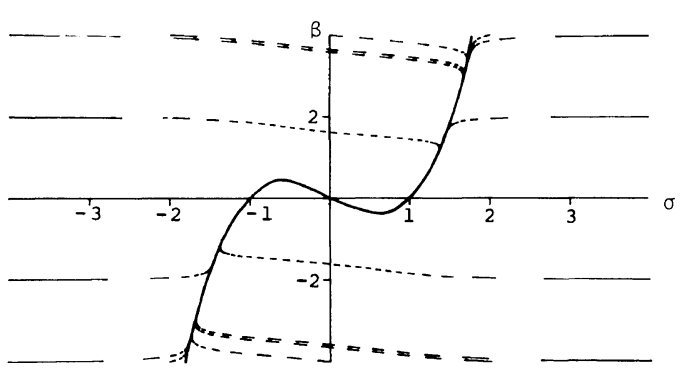

(a)

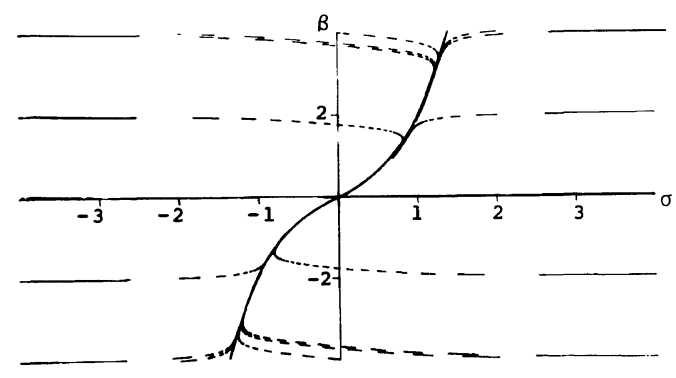

(b)

Figure 2. Similar trajectories to Figure 1 due to the catastrophe model' (10) instead of eq 2 . Selection is made with $M=\tau=1, \tau^{\prime}=5, A=-1$ (a), and $A=1$ (b) are selected. The minima of the pseudo-potentials are also shown with solid lines. 


\section{R. CHÛjô and K. TsuYamA}

these potential minimum curves with the abscissa axis. The situation in the case of $A<0$ is not so different from that in Figure 1, because the intersection is uniquely determined. On the contrary, the situation is unusual in the case of $A>0$. There are three intersections. Among these, one appearing at the origin is not a minimum but a maximum in this case. The remaining two are minima. The system tends to one of these two minima depending on the initial condition. This is the nonlinearity which is newly introduced by the application of catastrophe theory. The model decribed by eq 10 will be called the catastrophe model. It is impossible, at the present, to clarify the physical meaning of the quantity $A$. Qualitatively, $A$ perhaps originates from the distortion of the pseudo-potential due to structural defects included in the system.

\section{STRESS RELAXATION AND APPARENT RELAXATION SPECTRA}

We will apply the catastrophe model, eq 10 , to stress-relaxation phenomena. Solving eq 10 for various values of $A$ under appropriate initial conditions, we obtain the time dependence of stress, that

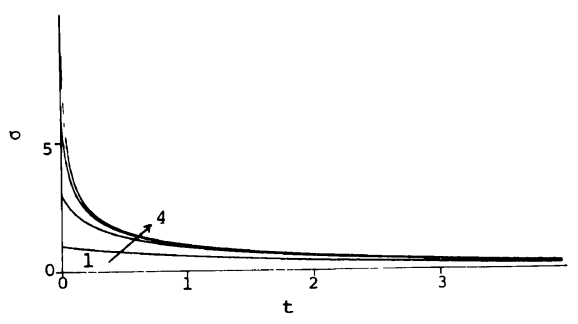

(a)

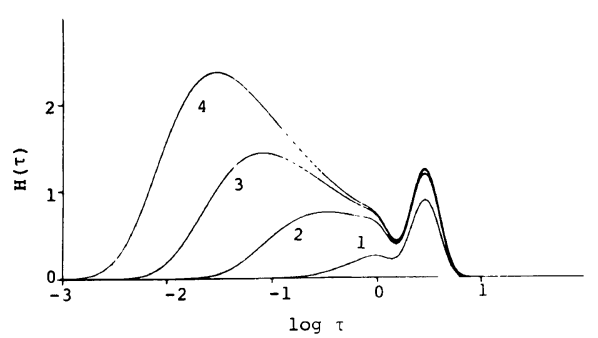

(b)

Figure 3. (a) Stress relaxation curves due to eq 10 and (b) their apparent relaxation spectra for $A=-1, \tau=3$, and $\beta=0,(1), 1,(2) 3,(3) 6$, and (4) 10 are selected as the initial values of $\sigma$. is, the stress-relaxation curve. In Figures $3 \mathrm{a}, 4 \mathrm{a}$, and $5 \mathrm{a}$ are shown the stress-relaxation curves for $A=-1$, 0 , and 1 , respectively. In these three figures, only the curves with $\tau=3$ and $\beta=0$ are reproduced from our data. The vanishing value of $\beta$ means that the strains are applied stepwide at $t=0$. This selection is due to the convenience of comparision with widely accepted curves in rheology. The values of the initial stress are noted in the figure captions for corresponding figures. In so far as the comparison between these three figures is concerned the sign of $A$ dose not affect seriously the stress-relaxation behavior. Comparing these figures with the stress-relaxation curve due to linear viscoelasticity, we can find a more drastic decrease in stress at an earlier stage of the relaxation.

As stated in the Introduction, we do not introduce the concept of relaxation spectra. Figures $3 a, 4 a$, and $5 \mathrm{a}$ are, indeed, the solution of eq 10 without the introduction of any kind of spectra. We will try to derive the apparent relaxation spectra for the stress relaxation in the above figures with the aid of a method commonly used in the analysis of linear viscoelasticity; the relaxation function is defined by

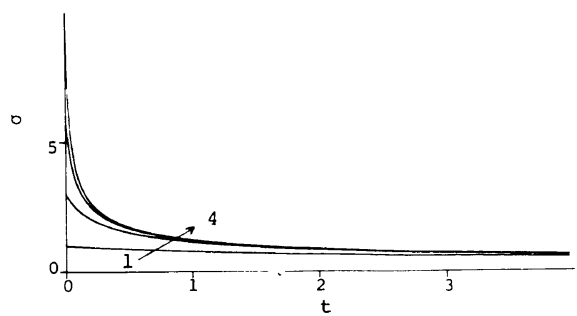

(a)

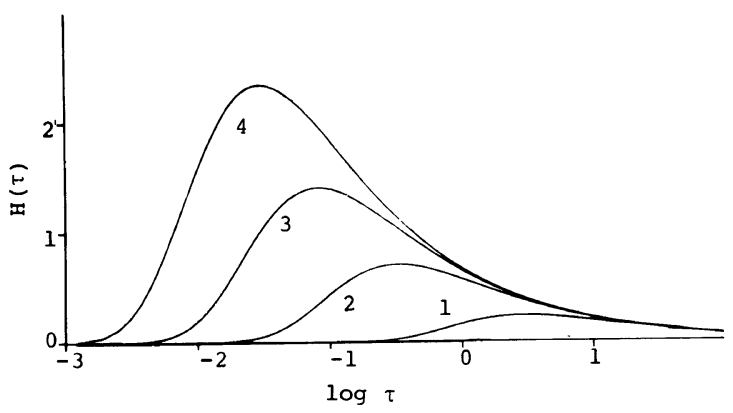

(b)

Figure 4. (a) Stress relaxation curves due to eq 10 and (b) their apparent relaxation spectra for $A=0, \tau=3$, and $\beta=0,(1), 1,(2) 3,(3) 6$, and (4) 10 are selected as the initial values of $\sigma$. 


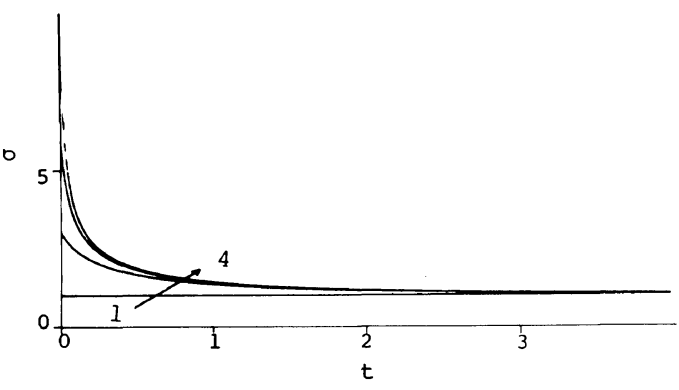

(a)

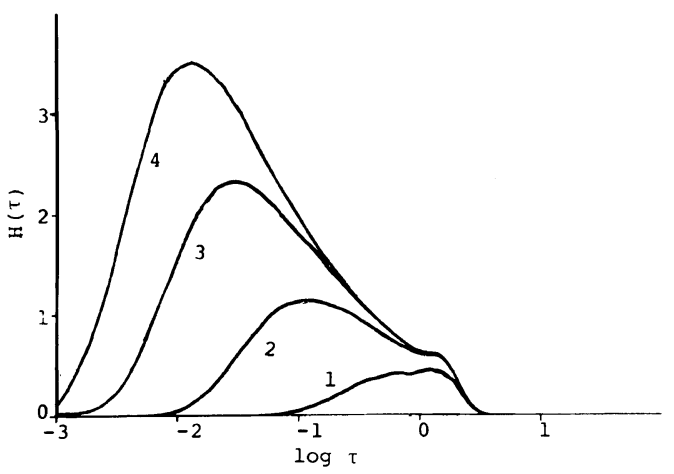

(b)

Figure 5. (a) Stress relaxation curves due to eq 10 and (b) their apparent relaxation spectra for $A=1, \tau=3$, and $\beta=0,(1), 1,(2) 3$, (3) 6, and (4) 10 are selected as the initial values of $\sigma$.

the ratio of $\sigma(t)$ to strain. For stepwise strain, $\sigma(t)$ itself can be used instead of the relaxation function. Therefore, $\sigma(t)$ is interrelated with $H(\sigma)$ by

$$
\sigma(t)=\int_{1-\infty}^{+\infty} \exp (-t / \tau) H(\tau) \mathrm{d} \ln \tau
$$

Putting $s=1 / \tau$ and $F(s)=(1 / s) H(1 / s)$, we have

$$
\sigma(t)=\int_{-\infty}^{+\infty} \exp (-t s) F(s) \mathrm{d} s
$$

we know this to be the Laplace transform. If $F(s)$ is bounded and continuous, $F(s)$ can be calculated by the inverstion formula ${ }^{12}$ of

$$
F(s)=\lim _{k \rightarrow \infty}(-1)^{k} /(k !)(k / s)^{k+1} f^{(k)}(k / s)
$$

where $f^{(k)}(x)$ means the $k$-th derivative of $f(x)$ with respect to $x$. In the course of numerical computation, we must inevitably truncate the operation of the lim up to a finite value of $k$. In the following discussion, we will truncate this operation up to the finite $k$ in which the relative error of $F(s)$ to that in $(k-1)$ st stage is less than $10^{-4}$.

In Figures $3 b, 4 b$, and $5 b$ are shown the apparent relaxation spectra obtained from the above procedure for the stress relaxations in Figures 3a, 4a, and $5 \mathrm{a}$, respectively. In spite of the absence of true relaxation spectra, we obtained apparent spectra as shown in Figures $3 b, 4 b$, and $5 b$. Although the precise shapes of these apparent spectra are different from those familiar in the linear viscoelasticity, namely, the sum of the wedge and box type spectra, we can say that there is the possibility of a derivation of apparent spectra. Comparing these apparent spectra with each other, we find that these are almost unimodal for $A=0$ and $A>0$, while these are bimodel for $A<0$. Furthermore, we must stress here that a successful derivation of apparent spectra for the nonlinear system which has nothing to do with spectra does not imply that relaxation spectra actually exist even if they are apparently derived. This is one point where we treated an essential nonlinear system instead of a linear or perturbed nonlinear system.

\section{APPLICATION TO SELF-OSCILLATING PHENOMENA}

As stated earlier, eq 10 with positive $A$ has two minima on the abscissa in Figure $2 \mathrm{a}$. Then, introducing a centripetal force on $\beta$ for this system, we have a self-oscillating system in which the oscillation is repeated between two minima. Zeeman ${ }^{13}$ introduced a similar centripetal force in order to analyze the relaxation of heartbeat. Following his procedure, we will introduce the centripetal force given by

$$
\mathrm{d} \beta / \mathrm{d} t=-\sigma / \tau^{\prime \prime}
$$

where $1 / \tau^{\prime \prime}$ is the proportionality constant defining the rate of $\beta$. The simultaneous solution of eq 10 and 14 are shown in Figure 6a for $A=\tau=\tau^{\prime \prime}=\sigma_{0}=\beta_{0}=1$. $\sigma_{0}$ and $\beta_{0}$ denote the initial values of $\sigma$ and $\beta$, respectively. In (2) and (3) of this figure are shown the time dependence of $\sigma$ and $\beta$, respectively. We could arrive at stress oscillation but not stress relaxation. In these figures the patterns of oscillation are considerably different from sinusoidal curves. This is a characteristic of nonlinear oscillating phenomena. With an increase in $A$, we can have more typically nonlinear oscillation. In (1) of Figure 6a is shown the trajectory of the solution on the $\beta-\sigma$ plane 

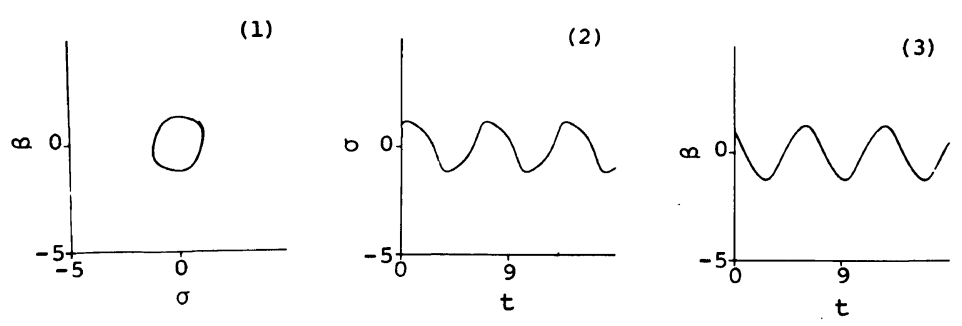

(a)
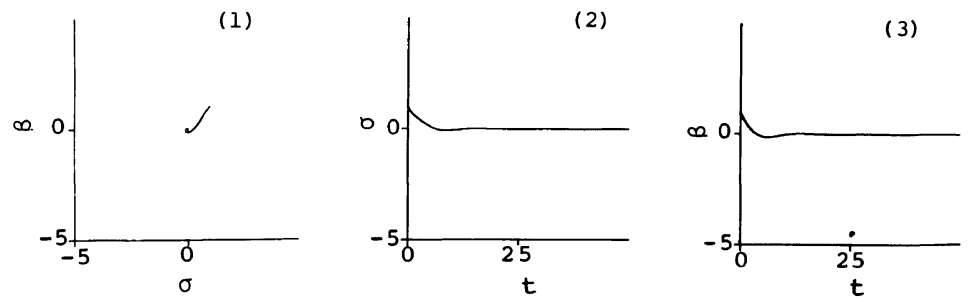

(b)

Figure 6. (a) Self-oscillating and (b) nonoscillating solutions of eq 10 and 14 for (a) $A=1$, (b) $A=-1$, and $\tau=\tau^{\prime \prime}=\sigma_{0}=\beta_{0}=1$. In both (a) and (b), (1) is the trajectory on $\beta-\sigma$ plane, (2) is the time dependence of $\sigma$, and (3) is the time dependence of $\beta$.

after the elimination of time from (2) and (3). This is a typical solution with a limit cycle. Then, if any perturbation is applied to this system, the system temporarily deviates from the limit cycle, but it finally goes back to the cycle, ensuring the stability of the self-oscillation described by eq 10 and 14 .

By selecting a negative value of $A$, we can never reach such a self-oscillating solution. In Figure $6 \mathrm{~b}$

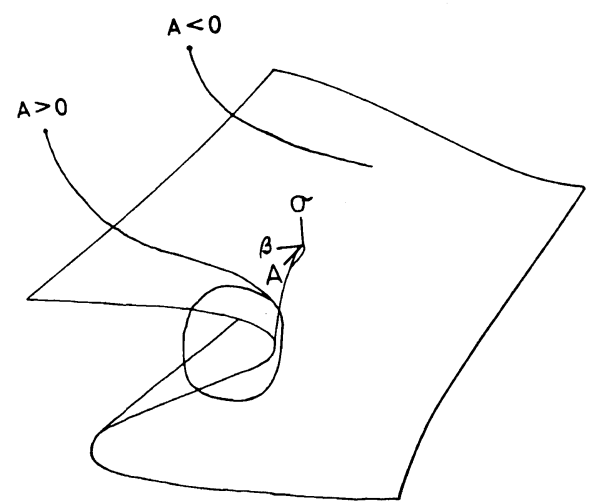

Figure 7. Schematical illustration of the behavior of the system described by eq 10 and 14 in $\beta-A-\sigma$ space. are shown similar plots to Figure $6 \mathrm{a}$ for the same selection of the parameter values except for $A=-1$. These are neither a self-oscillating nor a limit cycle solution.

In addition to eq 10 and 14 we must consider solving eq 4. After the accomplishment of the rapid relaxation due to eq 10 , the slow relaxation is performed on the pseudo-potential derived from the right-hand side of eq 10 . Such a slow relaxation is, of course, described by eq 4 . In so far as being confined to the trajectory of slow relaxation is concerned necessary, since only eq 10 and 14 participate in deciding the trajectory. This is the reason eq 4 is not considered in the discussion of Figures $6 \mathrm{a}$ and $6 \mathrm{~b}$.

The behavior in the $\beta-A-\sigma$ space is schematically illustrated in Figure 7. A family of trajectories for slow relaxation is given by a manifold with the shape of a furrow, whose projection on $\beta-A$ plane is cuspshaped. We can say that self-oscillation for positive $A$ is due to so-called delay convention. ${ }^{10}$

\section{APPLICATION TO DRAW RESONANCE PHENOMENA}

There are self-oscillating phenomena in melt spin- 
ning and film formation processes which are called draw resonance and surging phenomena, respectively. We will apply the procedure in the preceding section to such oscillating phenomena. In order to apply this procedure, a minor modification is required.

Immediately after the discharge from the orifice, the flow of polymer melt is inflated to the direction perpendicular to the flow. This phenomenon is known as the Barus effect. We will assume the proportionality of the rate of input and/or the output of the melt into and/or from this inflated region to the rate of strain, namely,

$$
\mathrm{d} m / \mathrm{d} t \propto \mathrm{d} \varepsilon / \mathrm{d} t=\beta
$$

Dividing the flow of melt into constant and intermittent flows as shown in Figure 8, we can write the diameter of filament as

$$
y=s+\beta_{-}
$$

where $s$ and $\beta_{-}$are the contribution from the constant and the intermittent flows, respectively. When $\beta$ is positive, the part of intermittent flow is stored in the inflated region, so $\beta_{-}=0$. When $\beta$ is negative, the part flows out from the inflated region, and $\beta_{-}$is equal to the absolute value of $\beta$. In short,

$$
\beta_{-}=(1 / 2)|(\beta-|\beta|)|
$$

irrespective of the sign of $\beta$. In eq 16 the effect of shrinkage is not taken into consideration. The proportionality constant is not explicitly written in

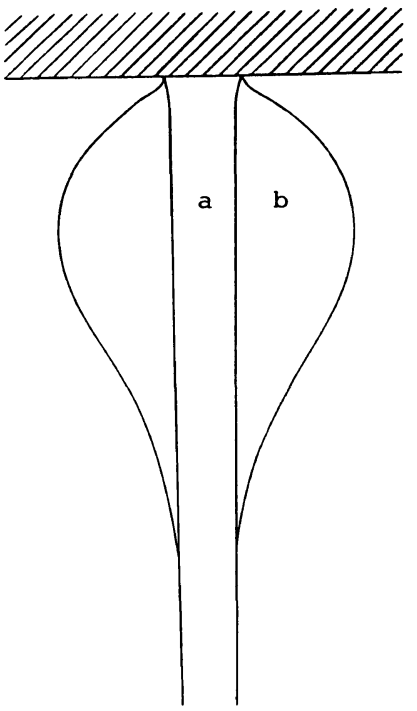

Figure 8. Division of the inflated region due to the Barus effect into (a) constant and (b) intermittent flows.

eq 16 , as yet.

We will compare the experimental results on the change of thickness of a polypropylene film along the film direction instead of the change of diameter of filament. These data were supplied by courtesy of Dr. S. Ichihara, according to whom the extruded melt can laminate on paper synchronously moving with the melt, and the measurement of the thickness becomes easier. In this case, deformation without the change of volume can be described in two dimen-

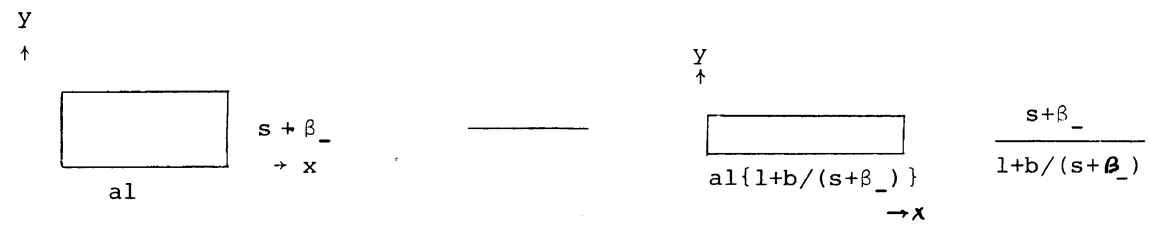

Figure 9. Schematical illustration of the dimension before and after drawing to $x$-direction.

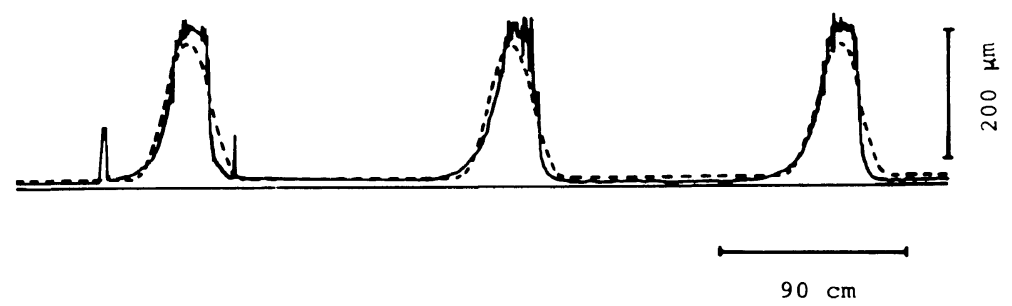

Figure 10. Comparison of the experimental (solid line) and the calculated (broken line) curves on the draw resonance of polypropylene. Scales for longitudinal and transverse directions are also shown. 
sional manner,

$$
\begin{gathered}
x=\Sigma a l\left\{1+b /\left(s+\beta_{-}\right)\right\} \\
y=\left(s+\beta_{-}\right) /\left\{1+b /\left(s+\beta_{-}\right)\right\}
\end{gathered}
$$

as shown in Figure 9. In these equations, $b$ is a proportionality constant and $a l$ is a scaling factor for length.

The solid line in Figure 10 is the record in a gauge meter measuring the thickness of polypropylene in which the temperature of the melt is $300^{\circ} \mathrm{C}$ and the take-up speed is $40 \mathrm{~m} \mathrm{~min}^{-1}$. We can find good agreement with the calculated curves of the broken line. In the broken line, the values of $a l, b$, and $s$ are selected as $0.123,0.550$, and 0.180 , respectively. Substituting eq 18 and 19 by these values and considering the independence of $a l$ and $b$ on the system condition, we can deduce that,

$$
s=0.18 /\left(0.5 A \tau / \tau^{\prime \prime}+0.5\right)
$$

In other words, the fraction of the constant flow decreases and that of the intermittent one increases with an increase of $A \tau / \tau^{\prime \prime}$. In order to illustrate this statement visually, the variations of $y$ with $x$ are shown in Figure 11 for various sets of parameter values. On comparison with the result of Ishihara and Kase, ${ }^{8}$ we can say that our treatment is better than theirs because of the adjustability in the selection of $A, \tau$ and $\tau^{\prime \prime}$. This advantage is due to the introduction of a elasticity term besides the pure viscosity one. Indeed, the calculated curve of Ishihara and Kase behaves similar to that in Figure 11 (2) which is far different from their experimental curve.

\section{CONCLUSION}

The catastrophe theory is certainly a powerful tool for describing nonlinear viscoelasticity. Especially, it is useful in understanding self-oscillating phenomena such as draw resonance. The catastrophe used here is the cusp catastrophe. Not restricted to viscoelasticity, our study suggests that the cusp catastrophe is applicable for analyzing nonlinear phenomena unsolved within the framework of the perturbation in other fields.

Numerical calculations were carried with the aid of a computer HITAC M-180 at the Information Processing Center, Tokyo Institute of Technology. Calculation results were plotted with the aid of Plotter WX 545.
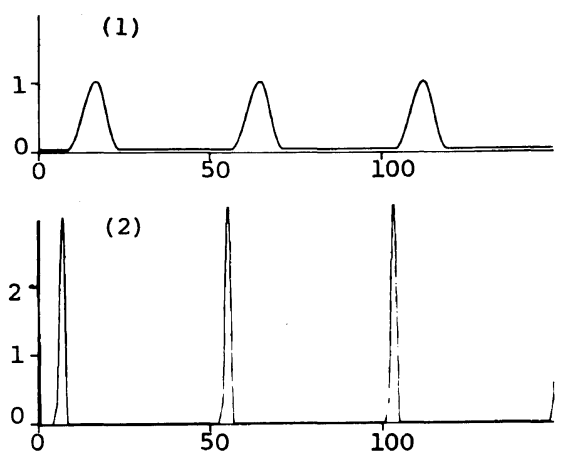

(3)

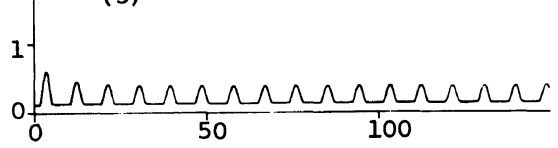

(4)

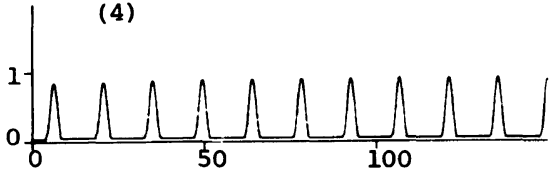

Figure 11. Examples of calculated curves corresponding to Figure 10 for various sets of parameter values. The abscissae are the length in arbitrary units and the ordinates are the diameter or thickness in arbitrary units. Parameter values are (1) $A=\tau=\tau^{\prime \prime}=1$, (2) $A=\tau=1$, $\tau^{\prime \prime}=0.1$, (3) $A=0.1, \tau=\tau^{\prime \prime}=0.25$, and (4) $A=0.1, \tau=1$, $\tau^{\prime \prime}=0.1$.

A lot of figures corresponding to Figures 2, 3, 6, 10 , and 11 with different parameter values are available in copy form.

Acknowledgements. The authors would like to express their sincere gratitude to Dr. N. Nakajima, B. F. Goodrich Chemical Company for his stimulating discussion. We are also grateful to Dr. S. Ichihara of Mitsubishi Petrochemical Company Limited for his kind supply of the experimental data on draw resonance. One of the authors (R.C.) wishes to dedicate this paper to Professor Nobuhiko Saito in honor of his 60 th birthday.

\section{REFERENCES}

1. For example, J. D. Ferry, "Viscoelastic Properties of Polymers," 2nd ed, John Wiley, New York, N.Y., 1970.

2. A. Odajima, J. Sohma, and M. Koike, J. Phys. Soc. Jpn., 12, 272 (1957). 


\section{Catastrophe Theory to Draw Resonance}

3. K. Fujimoto and T. Nishi, J. Soc. Rubber Ind. Jpn., 34, 828 (1972).

4. K. Fujimoto, T. Nishi, and R. Kado, Polym. J., 3, 448 (1972).

5. Y. Inoue, A. Nishioka, and R. Chûjô Makromol. Chem., 168, 163 (1973).

6. T. Matsumoto, Y. Segawa, Y. Warashina, and S. Onogi, Trans. Soc. Rheol., 17, 47 (1973).

7. V. Volterra, "Leçon sur la Théorie Mathématique de la Lutte pour la Vie," Gauthier-Villars, Paris, 1931.
8. H. Ishihara and S. Kase, J. Appl. Polym. Sci., 20, 169 (1976).

9. R. J. Fischer and M. M. Denn, Appl. Polym. Symp., 27, 103 (1975).

10. R. Thom, "Stabilité Structurelle et Morphogénèse," W. A. Benjamin Inc., Menlo Park, Calif., (1972).

11. J. M. T. Thompson and P. A. Shorrock, J. Mech. Phys. Solids, 23, 21 (1975).

12. G. Doetsch, "Theorie und Anwendung. der LapalaceTransformation", Springer, Berlin, (1973).

13. E. C. Zeeman, Towards Theor. Biol., 4, 8 (1972). 